\title{
A Study on Internet of Things Service Server Application for Provision of Automatic Service
}

\author{
Am-Suk Oh \\ Dept. of Digital Media Engineering, Tongmyong University, Busan, Korea \\ asoh@tu.ac.kr
}

\begin{abstract}
This paper researches about maker kit that surpasses development kit used for the development of existing simple firmware. The system is maker kit that easily derives idea and can realize it based on internet of things. The system consists of IoT-Kit, IoT-Kit Cloud Server, IoT-Kit App and IoT-Kit API. The system can understand all structures of internet of things service based on oneM2M standard platform.
\end{abstract}

Keywords: Internet of Things, MQTT, Arduino, Maker Movement, OneM2M

\section{Introduction}

'Maker Movement', the movement of open source manufacturing industry that makers share how to make products, has spread. Accordingly, the government of Korea has enlarged the political support for maker space establishment and IoT DIY education and supply [1-2].

However, the activation of maker culture is still incomplete because of low accessibility and the lack of professional power that it simply leans too much towards maker space establishment and operation [3-4].

This paper, therefore, researches about IoT maker kit that utilizes MQTT protocol for the interlock of Arduino-based practical service for makers, and tries to design and implement IoT platform server and maker client.

\section{System Configuration}

This paper researches about maker kit based on oneM2M standard IoT platform, beyond development kit for existing simple firmware development internship. Moreover, IoT maker kit that easily deduct and realize ideas through the understanding of the whole service structure will researched.

The system structure of IoT maker kit is as Figure 1. IoT-Kit in Figure 1 refers to internet of things maker board, IoT-Kit Cloud Server refers to IoT service drive server and IoT-Kit App refers to IoT service providing App.

Received (May 15, 2018), Review Result (July 5, 2018), Accepted (July 11, 2018) 


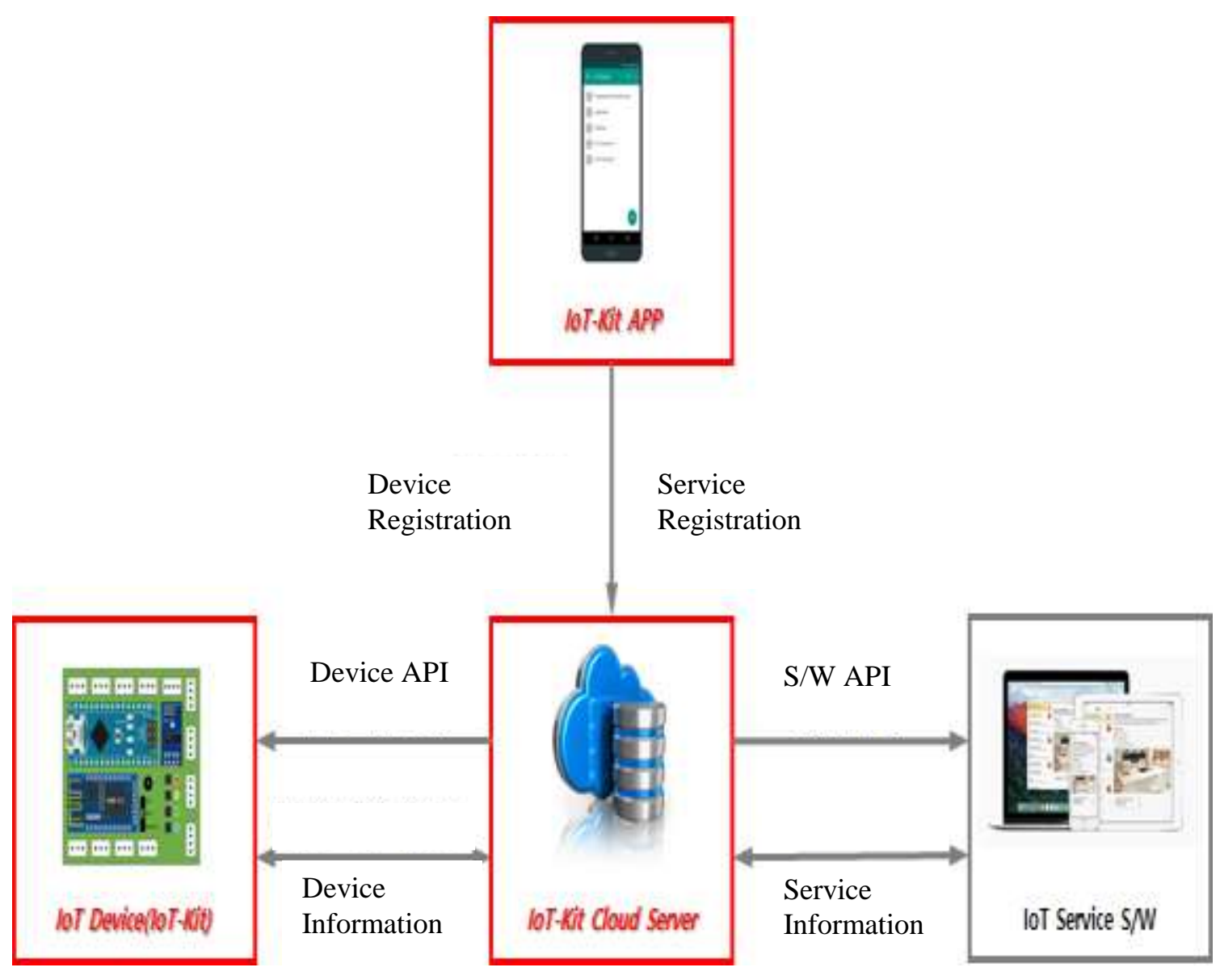

Figure 1. IoT Maker Kit Configuration

\section{IoT Maker Board}

\subsection{IoT Maker Board}

Figure 2 is a schematic diagram of IoT maker board hardware and has following functions.

- Equipped with WiFi and Bluetooth module based on Nano board from Arduino Uno and provides four shields for each digital, analog and I2C.

- Interlock with diverse sensors and interface module 


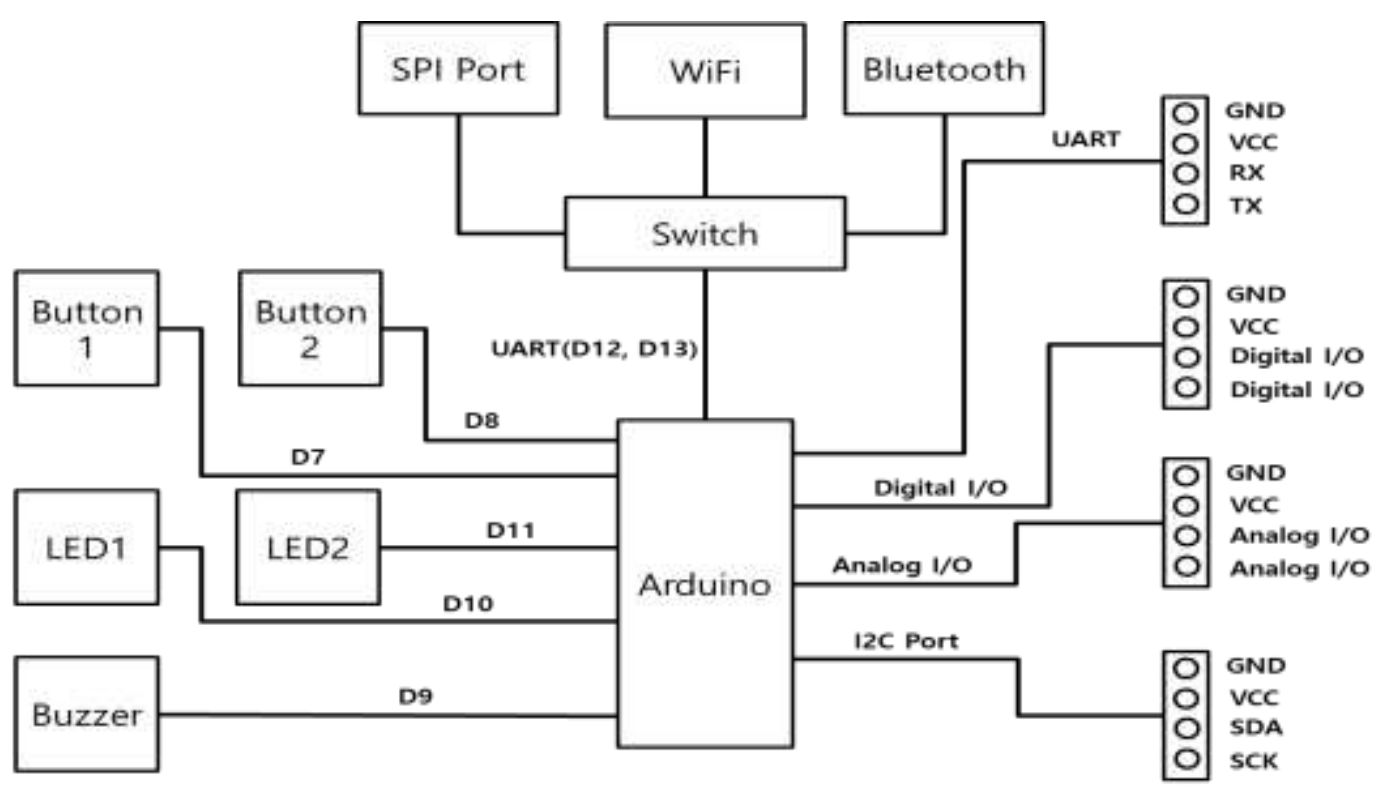

Figure 2. Hardware Diagram to Internet of Things Maker Board

\subsection{IoT-KIT Development Board}

Figure 3 is IoT-KIT development board and functions are as follows.

- Shield type of IoT communication board that is mountable on the most generalized Arduino Uno board

- Simplification of sensor module interface of existing IoT-KIT Mother Board: provides 4 interface of each Digital I/O, Analogue I/O and I2C

- Does not interface existing WiFi and Bluetooth module of IoT-KIT motherboard to gender board, but constructs circuit by settling module itself

- Provides module to UART data pin so that users can do porting firmware of WiFi, Bluetooth module on their own as the module is settled.

- Addition of board reset button for communication disruptions and initialization

- Addition of SMD LED to check the state of power and communication of WiFi, Bluetooth without special firmware.

- Consists of double connector for short prevention when equipped with Arduino Uno board as shield form.
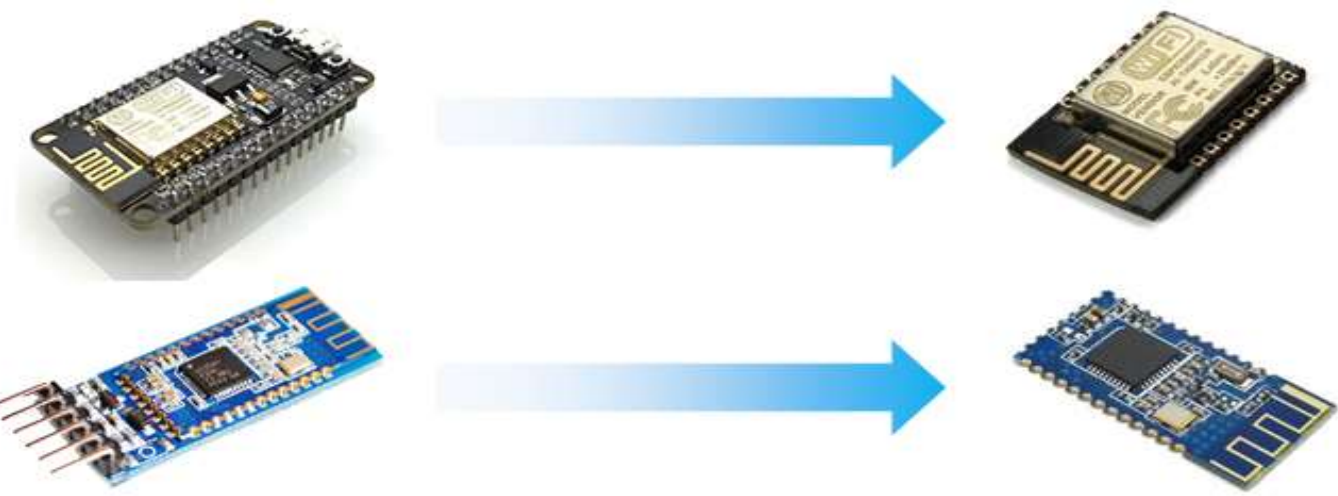

Figure 3. IoT-KIT Development Board 


\section{IoT Service Server}

\subsection{Service Server Module Constitution}

Figure 4 is a diagram of service server module and the functions are below.

- Router Module: module that conducts applicable functions according to the API request of IoT-Kit APP and responds result information.

- Authority Module: module that verifies user rights about information received through API or Socket communication from IoT-Kit APP.

- Socket Module: module that provides MQTT service between IoT-Kit server platform and APP.

- SQL Module: module that conducts query such as DB inquiry, enrolment according to the request of IoT-Kit APP.

- MQTT Module: module that conducts Publish/Subscribe functions, connected with (MQTT Broker).

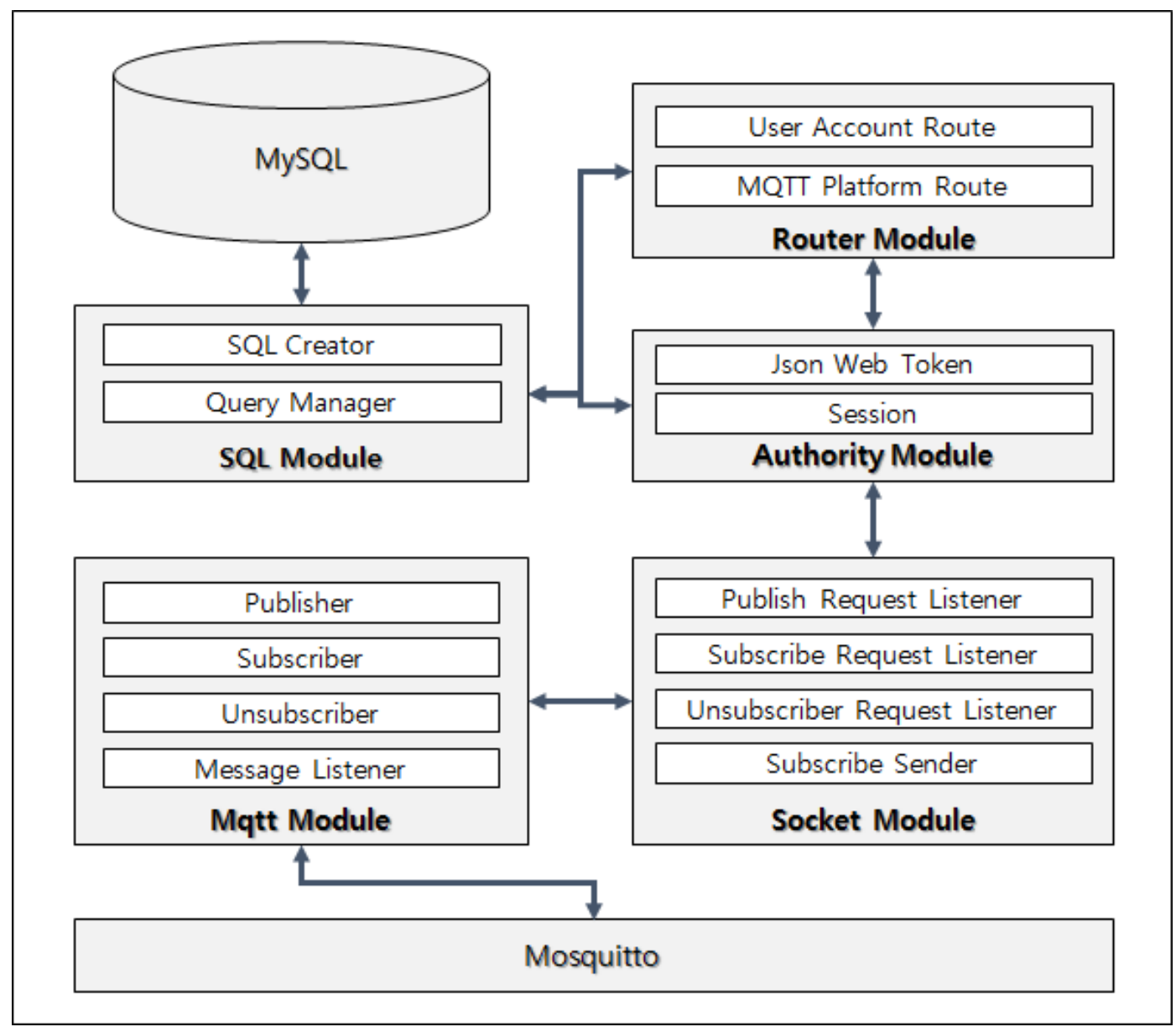

Figure 4. Service Server Module Configuration

\subsection{Service Server Function Test}

Output screen of server console is as figure 5 and its functions are below.

- Carries out Subscribe/Publish functions after user login through Login API. 
- Checks socket connection of web service server and client during MQTT service request of client.

- Checks conducting results of applicable functions according to Subscribe/Publish requests.

- Means the function performed normally if the result code is 1001 .

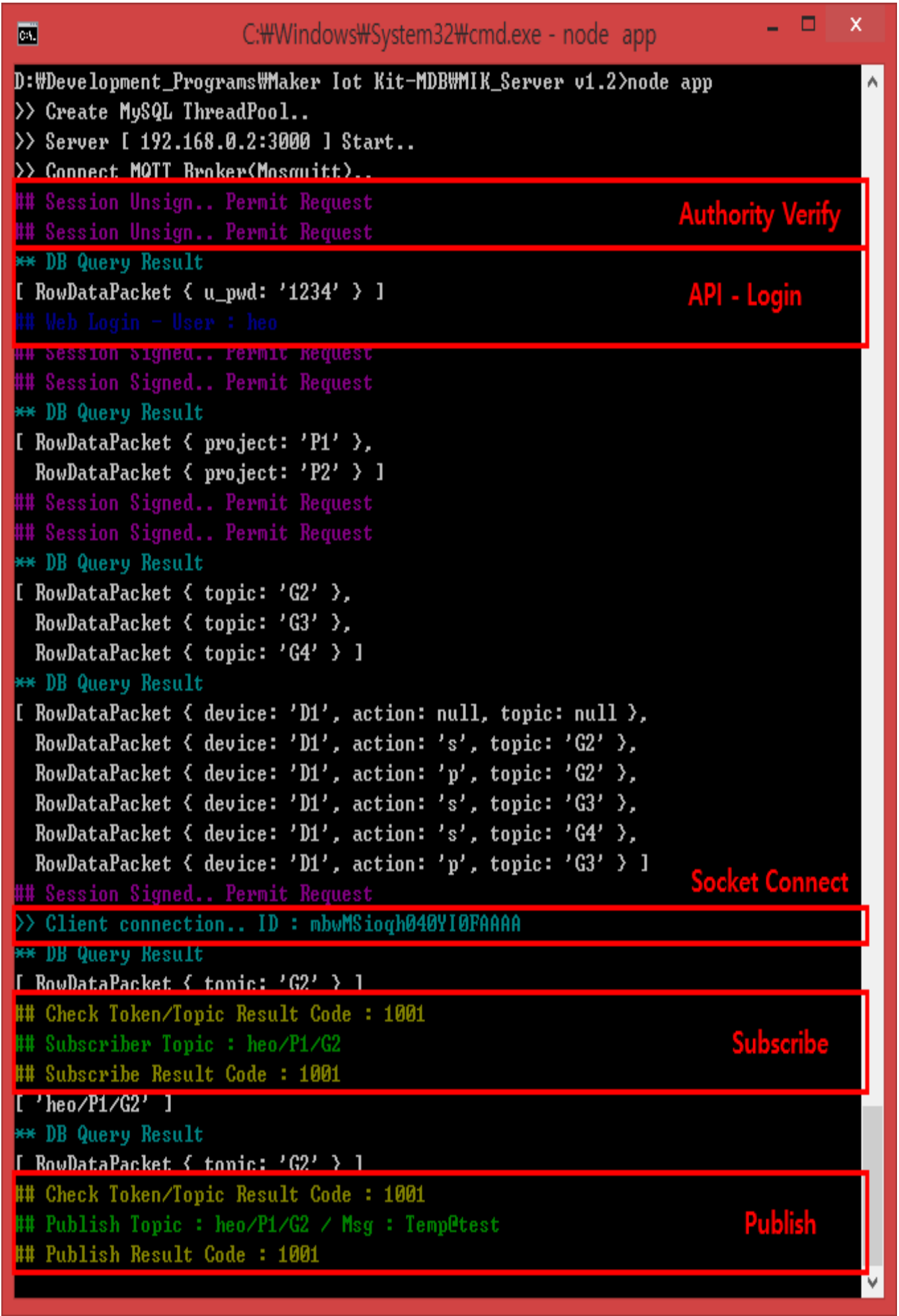

Figure 5. Server Console Output Screen 
Output screen of server console is as Figure 5 and its functions are below.

MQTT Broker console output screen is as Figure 6 and the functions are as follows.

- Web service server and MQTT Broker connect and conduct Subscribe/Publish.

- Checks the Subscribe/Publish function performance according to the service server request.

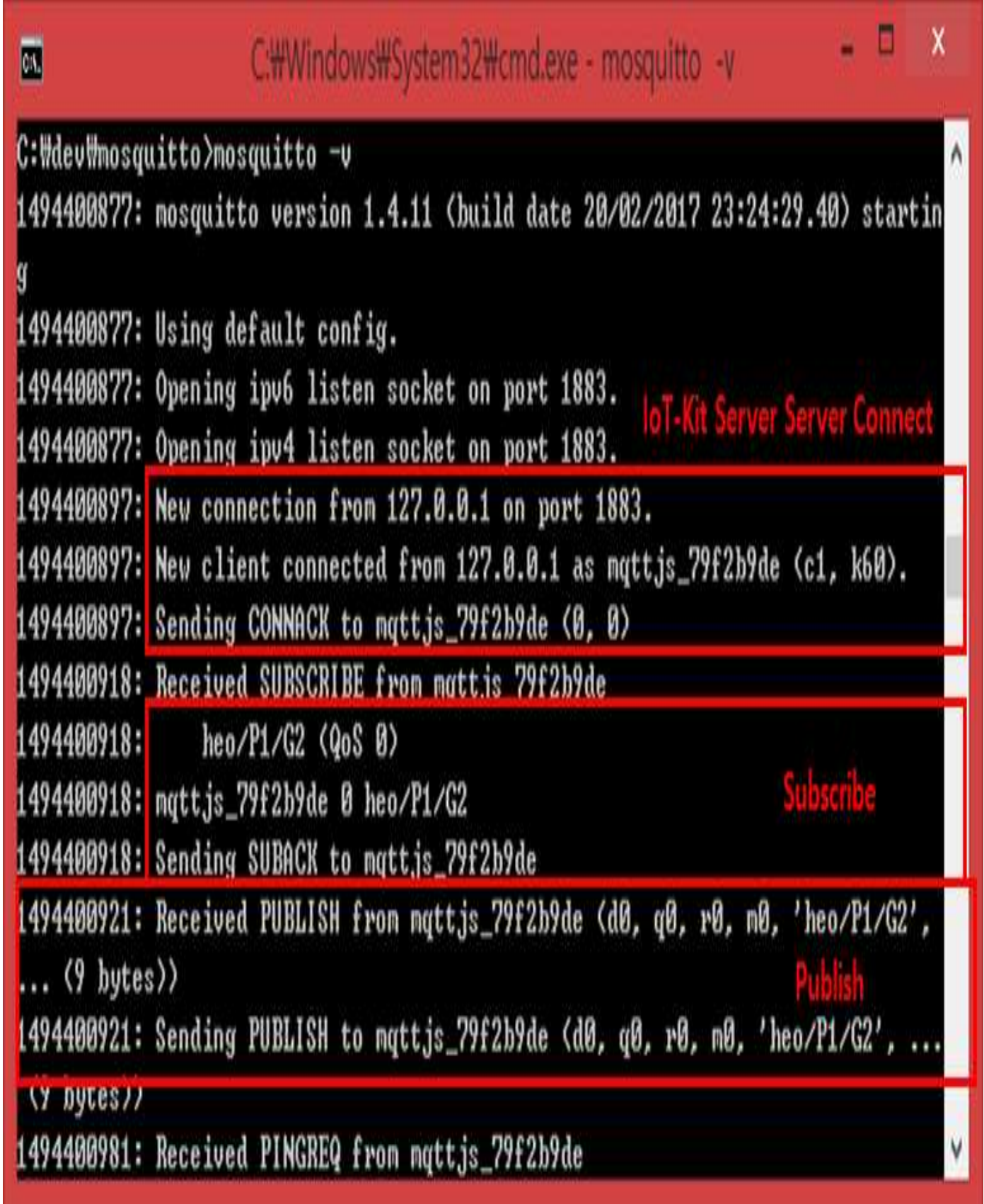

Figure 6. MQTT Broker Console Output Screen

\section{IoT Service Application}

\subsection{User Project Management Page}

- Settings page for the use of MQTT functions through Kit Platform 
- Consists of projects, topics, device stage by stage to prevent data sending/receiving error caused by the same topic among users.

- After login, users use MQTT functions through project subtopic and device settings.

- User Project Management enrolment procedure

1. Login through user ID/password

2. Project generation

3. Choosing project to use and generating topic to use in applicable projects

4. Enrolling topic to use for device generating Publish/Subscribe

\subsection{MQTT Publish/Subscribe Management Page}

- provides Publish and Subscribe about topic that user enrolled in web browser environment

- During Publish, sends applicable topics to Subscribed client by designating name of device as IoT Platform arbitrarily

- During web page load, progresses Subscribe by combining user ID, project, topic data altogether.

The result of IoT service application implementation is as follows.
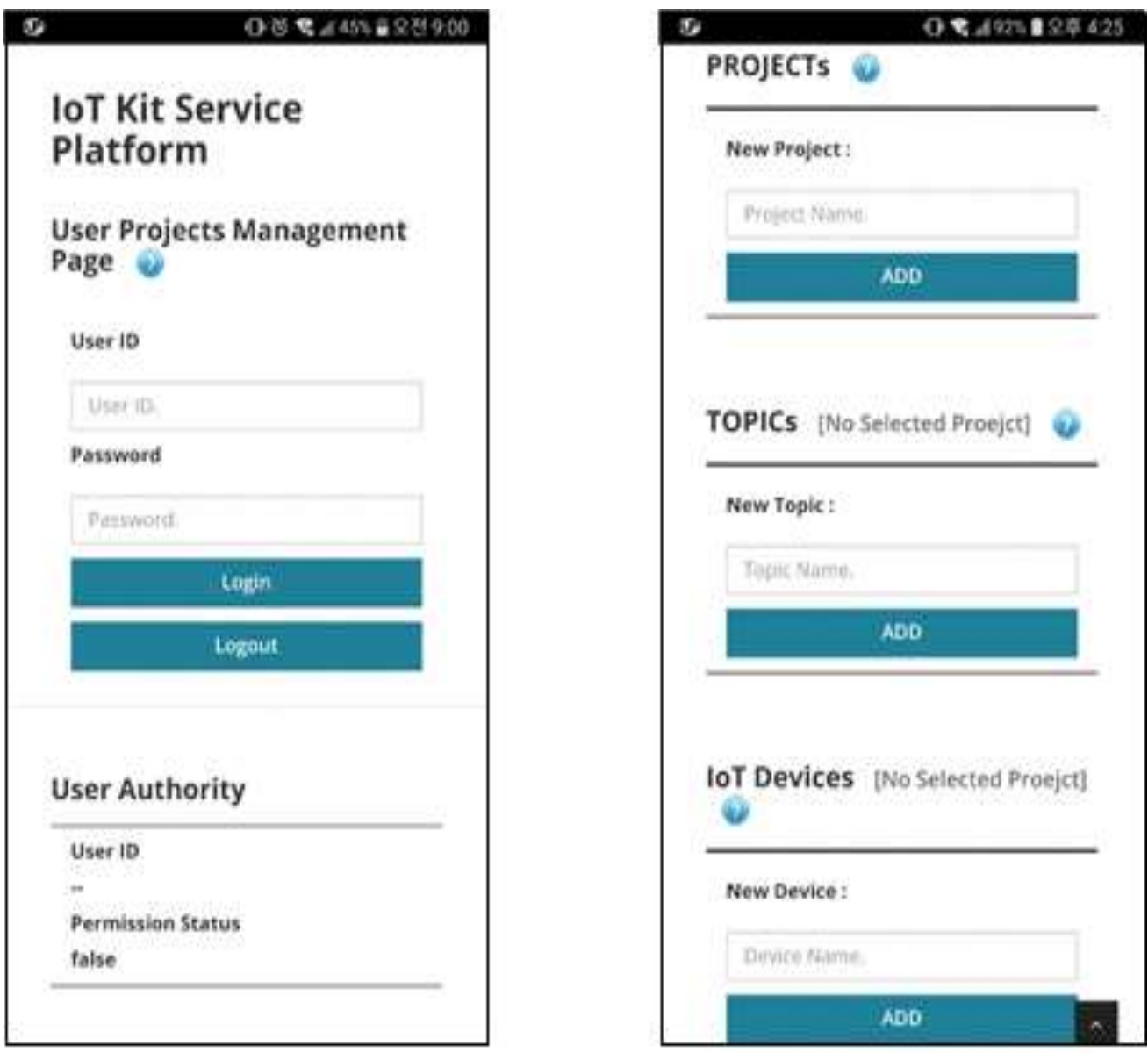

Figure 7. Service Application 


\section{Conclusion}

For maker, this thesis designed and implemented IoT maker board, IoT platform server and maker client that is capable of effective service interlocking that based on Arduino. IoT maker board libraries firmware to use WiFi module and Bluetooth module and developed to link IoT service. Furthermore, it implemented for users to utilize IoT platform server through Android and C\# library. Accordingly, it expected that the implementation of IoT service is possible in not only simple programming education and regardless of software and hardware developer.

\section{Acknowledgments}

This research supported by the Tongmyong University Research Grants 2018.

\section{References}

[1] S.-H. Kim, D.-H. Kim, H.-S. Oh, H.-S. Jeon and H.-J. Park, "The Data Collection Solution Based on MQTT for Stable IoT Platform", Journal of the Korea Institute of Information and Communication Engineering, vol. 20, no. 4, (2016).

[2] IBM Available: http://www.ibm.com/support/knowledgecenter/ko/SSCGGQ_1.2.0/com.ibm.ism.d oc/Planning/ov00102_.html.

[3] R. Cohn and R. Coppen, "OASIS Message Queuing Telemetry Transport (MQTT) TC[Internet]", Available:https://www.oasis-open.org/committees/tc_home.php? wgab brev =mqtt.

[4] S.-Y. Kim and K.-Y. Kim, "Standardization of IoT Service Platform", Communications of the Korean Institute of Information Scientists and Engineers, vol. 32, no. 6, (2014).

[5] S. H. Kim, D. H. Kim, H. S. Oh, H. S. Jeon and H. J. Park, "The Data Collection Solution Based on MQTT for Stable IoT Platforms", Journal of the Korea Institute of Information and Communication Engineering, vol. 20, no. 4, (2016) April, pp. 728-738.

[6] S. H. Shim and H. B. Kim, "Internet of Things and MQTT Technology", Korea Institute of Information Security and Cryptology, vol. 24, no. 6, (2014) December, pp. 37-47.

[7] S. Y. Kim, Y. J Jung and Y. S Hwang "Real-time and Parallel Semantic Translation Technique for Large-Scale Streaming Sensor Data in an IoT Environment”, Korea Information Science Society, vol. 42, no. 1, (2015) January, pp. 54-67.

[8] D. H. Kim and J. Kwak, "Design of Improved Authentication Protocol for Sensor Networks in IoT Environment", Journal of the Korea Institute of Information Security \& Cryptology, vol. 25, no. 2, (2015) April, pp. 467-478.

[9] W. Y. Uhm, K. K. Ryu and S. C. Kim, "A 94-GHz Phased Array Antenna Using a Log-Periodic Antenna on a GaAs Substrate", Journal of information and communication convergence engineering, vol. 13, no. 2, (2015), pp. 81-85.

[10] Y. H. Kim, "Maximum Likelihood (ML)-Based Quantizer Design for Distributed Systems", Journal of information and communication convergence engineering, vol. 13, no. 3, (2015), pp. 152-158.

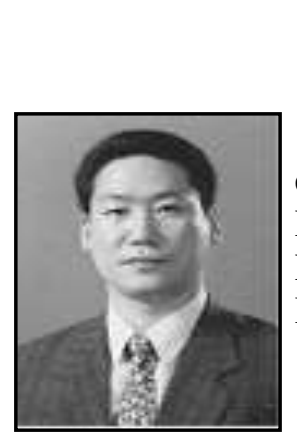

\section{Author}

Am-Suk Oh, he received Ph.D degree at the computer engineering of Pusan National University. He is currently with the Department of Media Engineering, Tongmyong University as Professor. His research interests are Healthcare System, Medical Information System, Big Data. 